Reprod. Nutr. Dévelop., 1986, 26 (2 B), 643-648.

\title{
Effet de la surrénalectomie sur la capacité de thermogenèse du tissu adipeux brun et le développement de l'obésité chez le rat Zucker fa/fa
}

\author{
R. BAZIN, S. KRIEF, Francine DUPUY, Marcelle LAVAU
}

INSERM U117, Institut Biomédical des Cordeliers

15, rue de l'Ecole de Médecine, 75270 Paris Cedex 06.

Summary. Effect of adrenalectomy on brown adipose tissue thermogenesis and development of obesity in Zucker fatty rats.

This study was undertaken to examine whether adrenalectomy performed during the weaning period could correct some of the first metabolic abnormalities to develop in obese $\mathrm{fa} / \mathrm{fa}$ rats : impaired thermogenesis in brown adipose tissue and hyperlipogenesis in interscapular brown and white (inguinal) adipose tissues.

Pups were adrenalectomized or sham-operated at 23 days of age and studied at 30 days of age. Body weight, interscapular brown adipose tissue and inguinal white adipose tissue weight were decreased after adrenalectomy in $\mathrm{Fa} / \mathrm{fa}$ and $\mathrm{fa} / \mathrm{fa}$ pups. Adrenalectomy had no effect on the thermogenic capacity of brown adipose tissue las assessed by GDP binding to mitochondria) which remained significantly lower in $\mathrm{fa} / \mathrm{fa}$ than in $\mathrm{Fa} / \mathrm{fa}$ rats. In both $\mathrm{Fa} / \mathrm{fa}$ and $\mathrm{fa} / \mathrm{fa}$ rats the lipogenic capacity of brown and white adipose tissues (as assessed by fatty acid synthetase activity) was dramatically reduced by adrenalectomy. However, in adrenalectomized rats, the fatty acid synthetase activity of brown and white adipose tissue remained 2 and 5 -fold higher, respectively, in $\mathrm{fa} / \mathrm{fa}$ than in $\mathrm{Fa} / \mathrm{fa}$ rats.

These results show that adrenalectomy at postweaning, did not affect specifically the rats bearing the fatty genotype but induced large alterations in both groups of rats. Adrenalectomized $\mathrm{fa} / \mathrm{fa}$ animals remained obese as compared to the appropriate controls.

\section{Introduction.}

Chez le rat Zucker fa/fa, l'hyperphagie est un facteur important du maintien de l'obésité (revue in Bray et York, 1979). Cependant, cette hyperphagie n'apparaît qu'au moment du sevrage (Stern et Johnson, 1977). A cet âge, bien que l'obésité ne soit pas encore visuellement détectable plusieurs anomalies pouvant la caractériser sont déjà installées, telles les augmentations de l'activité de la lipoprotéine lipase (Gruen et al., 1978 ; Boulangé et al., 1979) et des enzymes de la lipogenèse dans le tissu adipeux inguinal (Bazin et al., 1982), l'augmentation de la lipogenèse dans le tissu adipeux brun (Bazin et al., 1983), la diminution de la température interne (Godbole et al., 1978 ; Planche et al., 1983) et la diminution de la capacité de thermogenèse du tissu adipeux brun (Bazin et al., 1984 ; York et al., 1984).

Plusieurs auteurs ont rapporté que lorsque l'obésité est déjà bien installée chez le fa/fa la surrénalectomie supprime I'hyperphagie et réduit l'obésité (Yuki- 
mura et al., 1978 ; Bray, 1982) tout en corrigeant le déficit de thermogenèse existant dans le tissu adipeux brun, tandis que l'injection de corticostérone annule tous les effets de cette manipulation (Holt et York, 1982).

Dans ce travail, nous avons étudié pendant la période qui suit immédiatement le sevrage, avant que l'obésité soit visuellemnt détectable, l'effet de la surrénalectomie sur quelques anomalies parmi les plus précoces chez le rat fa/fa : l'augmentation de la capacité de lipogenèse des tissus adipeux brun interscapulaire et blanc inguinal et la diminution de la capacité de thermogenèse du tissu adipeux brun.

\section{Matériel et Méthodes.}

Les rats Zucker sont issus de notre propre élevage par croisements de mâles homozygotes obèses ( $\mathrm{fa} / \mathrm{fa}$ ) et de femelles hétérozygotes non obèses ( $\mathrm{Fa} / \mathrm{fa}$ ). A l'âge de 16 jours l'identification du génotype des rats est faite selon une technique déjà décrite (Lavau et Bazin, 1982), après prélèvement chirurgical d'un pannicule de tissu adipeux inguinal. Les animaux sevrés à 18 jours subissent une surrénalectomie (simulée chez les témoins) à 23 jours puis sont sacrifiés à 30 jours entre $9 \mathrm{~h}$ et $10 \mathrm{~h}$. L'activité de la synthétase des acides gras est mesurée par une technique spectrophotométrique (Halestrap et Denton, 1973) dans les surnageants (105000xg) des homogénats de tissus. Les lipides totaux sont mesurés dans les tissus par gravimétrie après extraction dans le chloroforme/méthanol $12: 1$, $\mathrm{vol} / \mathrm{vol}$ ). Dans les mitochondries du tissu adipeux brun isolées selon la méthode de Slinde et al., (1975), l'activité de la cytochrome c oxydase est mesurée par polarographie (Schnaitman et al., 1967) et la liaison du GDP à I'aide de GDP tritié selon la technique de Nicholls (1976) avec les modifications précédemment décrites (Bazin et al., 1984). La corticosteronémie est mesurée dans le serum par un dosage radioimmunologique utilisant un anticorps anticorticostérone-21 thyroglobuline (Miles Laboratories).

Les protéines sont déterminées par la méthode de Bradford (1976). L'analyse statistique des résultats est faite par comparaison de groupes à l'aide du test $t$ de Student.

\section{Résultats.}

Une semaine après la surrénalectomie les rats $\mathrm{fa} / \mathrm{fa}$ et $\mathrm{Fa} / \mathrm{fa}$ de 30 jours présentent un important retard de croissance. On observe une diminution de $20 \%$ du poids corporel $(44,5 \pm 1,3 \mathrm{~g}$ et $46,3 \pm 1,47 \mathrm{~g}$ chez les $\mathrm{Fa} / \mathrm{fa}$ ( $\mathrm{n}=17$ ) et les $\mathrm{fa} / \mathrm{fa}(\mathrm{n}=20)$ surrénalectomisés contre $54,8 \pm 1,8 \mathrm{~g}$ et $60,3 \pm 2,6 \mathrm{~g}$ chez les $\mathrm{Fa} / \mathrm{fa}(\mathrm{n}=22)$ et les $\mathrm{fa} / \mathrm{fa}(\mathrm{n}=19)$ témoins. A 30 jours, il n'y a pas de différence de corticostéronémie entre les deux génotypes $(23 \pm 2,2$ et $21 \pm 1,7 \mu \mathrm{g} / 100 \mathrm{ml}$ respectivement chez les $\mathrm{Fa} / \mathrm{fa}(\mathrm{n}=22)$ et les $\mathrm{fa} / \mathrm{fa}$ ( $\mathrm{n}=19$ ), elle $\mathrm{n}^{\prime}$ est plus détectable chez les animaux surrénalectomisés (seuil de détection : $0,5 \mu \mathrm{g} / 100 \mathrm{ml}$ ) . 
Les résultats présentés dans la figure 1 montrent que le poids de 1 pannicule de tissu adipeux blanc inguinal est 3 fois plus important chez les animaux $\mathrm{fa} / \mathrm{fa}$ comparés aux $\mathrm{Fa} / \mathrm{fa}$. La surrénalectomie diminue considérablement le poids de ce tissu chez les obèses comme chez les non-obèses, cependant le tissu des obèses reste beaucoup plus développé que celui des $\mathrm{Fa} / \mathrm{fa}$ ( 4 fois). La masse de tissu adipeux brun interscapulaire, deux fois plus importante chez les obèses que chez les non-obèses, est très affectée par la surrénalectomie $(-30 \%$ et $-50 \%$ respectivement chez les $\mathrm{Fa} / \mathrm{fa}$ et les $\mathrm{fa} / \mathrm{fa}$ ). La figure 1 montre également que la surrénalectomie diminue la teneur en lipides du tissu adipeux brun dans les mêmes proportions chez les $\mathrm{Fa} / \mathrm{fa}$ et les $\mathrm{fa} / \mathrm{fa}$.

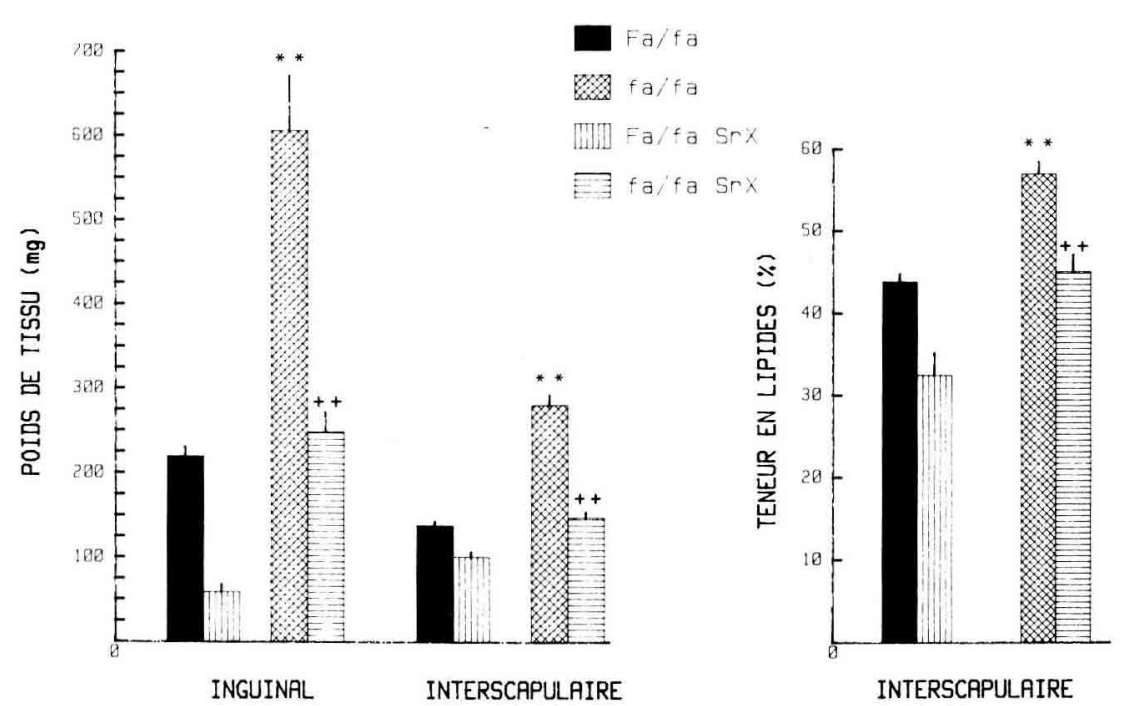

FIG. 1. - Poids des tissus adipeux blanc et brun et teneur en lipides du tissu adipeux brun chez les rats Zucker de 30 jours, normaux et surrénalectomisés (SrX).

${ }^{*} \mathrm{P}<0,01 \mathrm{fa} / \mathrm{fa}$ contre $\mathrm{Fa} / \mathrm{fa} ;++\mathrm{P}<0,01 \mathrm{fa} / \mathrm{fa} \mathrm{SrX}$ contre $\mathrm{Fa} / \mathrm{fa} \mathrm{SrX}$. Nombre d'animaux : $17,22,12 \mathrm{Fa} / \mathrm{fa} ; 13,19,7 \mathrm{fa} / \mathrm{fa} ; 11,17,7 \mathrm{Fa} / \mathrm{fa} \mathrm{SrX} ; 15,20,10 \mathrm{fa} / \mathrm{fa} \mathrm{SrX}$ pour les mesures respectives des poids de tissu blanc inguinal, brun interscapulaire et de la teneur en lipides du tissu brun.

La figure 2 présente la capacité lipogénétique des tissus adipeux brun et blanc mesurée par l'activité de la synthétase des acides gras (SAG). Chez les animaux témoins obèses comparés aux non-obèses, l'activité de la SAG est 3 et 4 fois plus importante respectivement dans les tissus adipeux brun et blanc. Dans les deux génotypes, la surrénalectomie diminue considérablement cette activité enzymatique $(-80 \%$ et $-90 \%$ dans le tissu brun, - $90 \%$ et $-85 \%$ dans le tissu inguinal, respectivement chez les $\mathrm{Fa} / \mathrm{fa}$ et les $\mathrm{fa} / \mathrm{fa}$ ).

Les résultats présentés dans le tableau 1 montrent que l'activité de la cytochrome c oxydase (marqueur de l'activité mitochondriale) est identique dans les deux génotypes et n'est pas modifiée par la surrénalectomie. Chez les animaux normaux, la capacité de thermogenèse mesurée par la liaison du GDP aux mitochondries du tissu adipeux brun est diminuée de $40 \%$ chez les fa/fa comparés 
avec les $\mathrm{Fa} / \mathrm{fa}$. La surrénalectomie ne restaure pas la capacité de thermogenèse déficiente chez les obèses.

\section{TABLEAU 1}

Capacité de thermogenèse du tissu adipeux brun.

\begin{tabular}{|c|c|c|c|c|}
\hline & $\mathrm{Fa} / \mathrm{fa}$ & $\mathrm{Fa} / \mathrm{fa} \mathrm{SrX}$ & $\mathrm{fa} / \mathrm{fa}$ & $\mathrm{fa} / \mathrm{fa} \operatorname{Sr} X$ \\
\hline $\begin{array}{l}\text { Cytochrome c oxydase } \\
\text { ( } \mu \text { mol. } 02 / \mathrm{min} \text { par } \mathrm{mg} \\
\text { prot. mitochondriales) }\end{array}$ & $5,3 \underset{(15)}{ \pm 0,31}$ & $4,9 \underset{(16)}{ \pm 0,20}$ & $4,8 \pm 0,20$ & $4,9 \underset{(16)}{ \pm 0,25}$ \\
\hline $\begin{array}{l}\text { GDP (pmol. par mg } \\
\text { prot. mitochondriales) }\end{array}$ & ${ }_{(22)}^{142 \pm} 14$ & $\underset{(17)}{138 \pm 14}$ & $\frac{90 \pm 6}{(19)^{\mathrm{a}}}$ & $\frac{105 \pm 5}{(20)^{b}}$ \\
\hline
\end{tabular}

Activité de la cytochrome oxydase et liaison du GDP dans des mitochondries isolées de tissu adipeux brun interscapulaire de rats Zucker témoins et surrénalectomisés ( $\mathrm{SrX}$ ).

a : $\mathrm{P}<0,01 \mathrm{fa} / \mathrm{fa}$ vs $\mathrm{Fa} / \mathrm{fa} ; \mathrm{b}: \mathrm{P}<0,05 \mathrm{fa} / \mathrm{fa} \mathrm{SrX}$ vs $\mathrm{Fa} / \mathrm{fa}$ Srx. Nombre d'animaux indiqué entre parenthèses.

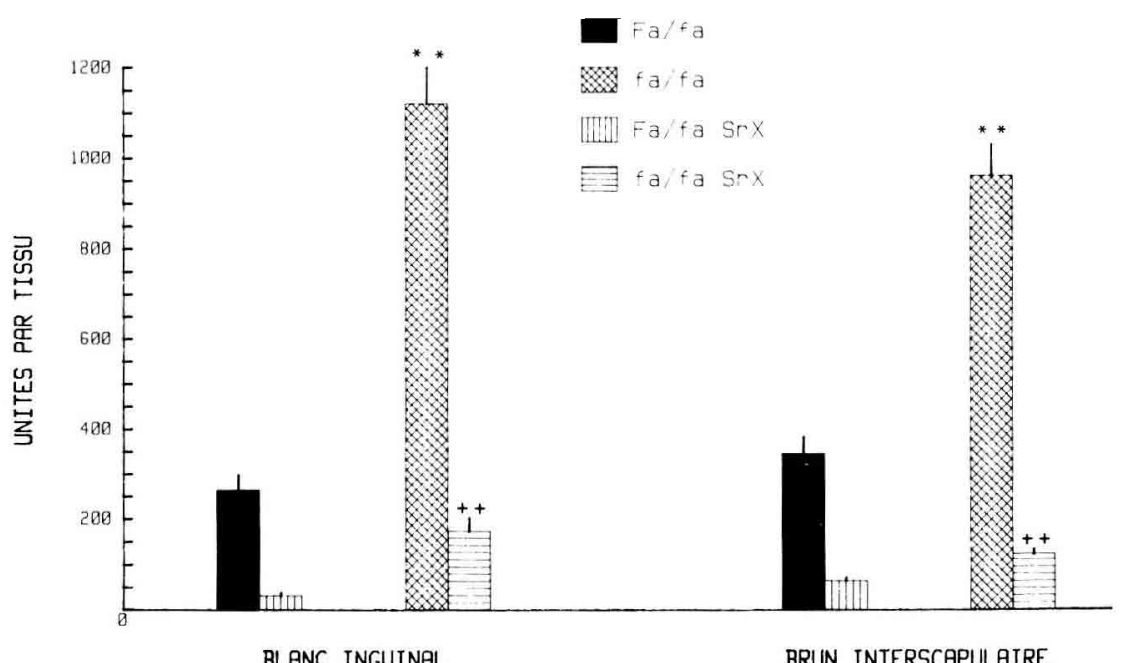

FIG. 2. - Activité de la synthétase des acides gras dans les tissus adipeux brun et blanc de rats Zucker de 30 jours, normaux et surrénalectomisés (SrX).

L'activité est représentée en unités par tissu. Une unité est définie comme la quantité d'enzymes nécessaire pour catalyser la formation de 1 nanomole de produit/min à $37{ }^{\circ} \mathrm{C}$. Nombre d'animaux : 17, $10 \mathrm{Fa} / \mathrm{fa} ; 13,12 \mathrm{fa} / \mathrm{fa} ; 10,10 \mathrm{Fa} / \mathrm{fa} \mathrm{SrX} ; 15,10 \mathrm{fa} / \mathrm{fa} \mathrm{SrX}$ pour la mesure de l'activité enzymatique respectivement dans le tissu blanc inguinal et brun interscapulaire.

\section{Discussion.}

Chez les animaux âgés de 30 jours, la corticostéronémie n'est pas différente entre les $\mathrm{Fa} / \mathrm{fa}$ et les fa/fa. Yukimura et al., (1978) ont rapporté un résultat identique chez les animaux de 8 à 10 semaines, tandis que York et al., (1984) ont noté au même âge une diminution de la concentration sérique de cette hormone chez les fa/fa comparés au Fa/fa. A l'âge de 12 semaines, Martin et al., (1978) ont rap- 
porté, à l'opposé, une corticostéronémie plus élevée chez les fa/fa que chez les $\mathrm{Fa} / \mathrm{fa}$.

Dans nos conditions expérimentales, la surrénalectomie pratiquée au moment du sevrage ne provoque pas un amaigrissement spécifique chez les obèses comme cela a été décrit chez les rats Zucker plus âgés (Yukimura et al., 1978 ; York et Godbole, 1979). Dans les deux génotypes, après surrénalectomie, la diminution considérable des réserves lipidiques que I'on observe dans le tissu adipeux blanc est cohéreñte avec la chute très importante observée dans l'activité lipogénétique de ce tissu. Cette étude montre que l'hyper-capacité de lipogenèse des tissus adipeux blanc et brun qui est une anomalie très précocement détectable chez le rat $\mathrm{fa} / \mathrm{fa}$ (Bazin et al., 1982, 1983) persiste après surrénalectomie. Ces résultats sont en désaccord avec ceux obtenus par York et Godbole (1979) montrant que, après surrénalectomie, la lipogenèse mesurée in vivo dans le tissu adipeux blanc et le foie, n'est pas changée chez les $\mathrm{Fa} / \mathrm{fa}$ mais diminue chez les $\mathrm{fa} / \mathrm{fa}$.

Dans le tissu adipeux brun des rats de 30 jours la capacité de thermogenèse altérée chez l'obèse n'est pas restaurée par la surrénalectomie comme cela a été rapporté par Holt et York (1982) chez le rat Zucker de 6 semaines.

Les différents travaux montrant que la surrénalectomie réduit spécifiquement certaines caractéristiques de l'obésité du rat fa/fa comparé au Fa/fa ont été réalisés sur des animaux âgés de 6 à 10 semaines qui présentent déjà une obésité considérablement amplifiée par l'hyperphagie. Notre travail montre que si la surrénalectomie est pratiquée de manière plus précoce, au moment du sevrage, elle altère notablement le développement de la capacité de lipogenèse des rats obèses comme de leurs frères non-obèses et ne permet pas de réactiver la thermogenèse déficiente dans le tissu brun de l'obèse.

En conc/usion, la surrénalectomie pratiquée dans la période du sevrage, chez les rats Zucker $\mathrm{Fa} / \mathrm{fa}$ et $\mathrm{fa} / \mathrm{fa}$, ne modifie pas le profil caractéristique que présente l'obèse quand on le compare à son témoin non-obèse.

11 Réunion du groupe Développement I.N.R.A. Montpellier, 22-24 mai 1985.

\section{Références}

BAZIN R., LAVAU M., 1982. Development of hepatic and adipose tissue lipogenic enzymes and insulinemia during suckling and weaning on to a high-fat diet in Zucker rat. J. Lipid Res., 23 , 839-849.

BAZIN R., LAVAU M., GUICHARD C., 1983. Development of fatty acid synthetic capacity in interscapular brown adipose tissue during suckling in genetically obese Zucker rats. Biochem. J., 216, 543-549.

BAZIN R., ETEVE D., LAVAU M., 1984. Evidence for decreased GDP binding to brown-adipose tissue mitochondria of obese Zucker $(\mathrm{fa} / \mathrm{fa})$ rats in the very first days of life. Biochem. J., 221, 241-245.

BOUlANGE A., PLANCHE E., de GASQUET P., 1979. Onset of genetic obesity in the absence of hyperphagia during the first week of life in the Zucker rat (fa/fa). J. Lipid Res., 20. 857-864.

Reproduction, Nutrition, Développement, $n^{\square} 2$ B-86. -9 
BRADFORD M. M., 1976. A rapid and sensitive method for the quantitation of microgram quantities of protein utilizing the principle of protein-dye binding. Anal. Biochem., 72, 248254.

BRAY G. A., 1982. Regulation of energy balance: studies on genetic, hypothalamic and dietary obesity. Proc. Nutr. Soc., 41, 95-108.

BRAY G. A., YORK D. A., 1979. Hypothalamic and genetic obesity in experimental animals : an autosomic and endocrine hypothesis. Physiol. Rev., 59, 719-809.

GODBOLE V., YORK D. A., BLOXHAM D. P., 1978. Developmental changes in fatty $(\mathrm{fa} / \mathrm{fa})$ rat : evidence for defective thermogenesis preceding the hyperlipogenesis and hyperinsulinemia. Diabetologia, 15, 41-44.

GRUEN R., HIETANEN E., GREENWOOD M. R. C., 1978. Increased adipose tissue lipoprotein lipase activity during the development of the genetically obese rat fa/fa. Metabolism, 27, 1955-1966.

HALESTRAP A. P., DENTON R. M., 1973. Insulin and the regulation of adipose tissue acetyl CoA carboxylase. Biochem. J., 132, 509-513.

HOLT S., YORK D. A., 1982. The effect of adrenalectomy on GDP binding to brown-adipose tissue mitochondria of obese rats. Biochem. J., 208, 819-822.

LAVAU M., BAZIN R., 1982. Inguinal fat pad weight plotted versus body weight as a method of genotype indentification in 16-day-old Zucker rats. J. Lipid Res., 23, 941-943.

MARTIN R. J., WANGSNESS P. J., GAHAGAN J. H., 1978. Diurnal changes in serum metabolites and hormones in lean and obese Zucker rats. Horm. Metab. Res., 10, 187-192.

NICHOLLS D. G., 1976. Hamster brown adipose mitochondria. Purine nucleotide control of the ion conductance of the inner membrane, the nature of the nucleotide binding site. Eur. J. Biochem., 62, 223-228.

PLANCHE E., JOLIFF M., de GASQUET P., LeLIEPVRE X., 1983. Evidence of a defect in energy expenditure in 7-day-old Zucker rat (fa/fa). Am. J. Physiol., 245, E107-E113.

SCHNAITMAN C. V., ERWIN G., GREENAWALT J. W., 1967. The submitochondrial localization of monoamine oxydase. J. Cell Biol., 62, 223-228.

SLINDE E., PEDERSEN J. I., FLATMARK T., 1975. Sedimentation of buoyant density of brown adipose tissue mitochondria of guinea pigs. Anal. Biochem., 65, 581-585.

STERN J. S., JOHNSON P. R., 1977. Spontaneous activity and adipose cellularity in the genetically obese Zucker rat (fa/fa). Metabolism, 26, 371-380.

YORK D. A., GODBOLE V., 1979. Effect of adrenalectomy on obese "fatty » rats. Horm. Metab. Res., 11, 646 .

YORK D., HOLT S., ROTHWELL N., STOCK M., 1984. Effect of age and gene dosage on brown adipose tissue of Zucker obese fa/fa rats. Am. J. Physiol, 246. E391-E396.

YUKIMURA Y., BRAY G. A., WOLFSEN A. R., 1978. Some effects of adrenalectomy in the fatty rat. Endocrinology, 103, 1924-1928. 